\title{
Epoxidized vegetable oils as processing aids and activators in carbon-black filled natural rubber compounds
}

\author{
Ganga Chandrasekara ${ }^{1}$, M.K. Mahanama ${ }^{2}$, D.G. Edirisinghe ${ }^{2}$ and L. Karunanayake ${ }^{1^{*}}$ \\ ${ }^{1}$ Department of Chemistry, Faculty of Applied Science, University of Sri Jayewardenepura, Gangodawila, Nugegoda. \\ ${ }^{2}$ Rubber Research Institute of Sri Lanka, Thelawala Road, Ratmalana.
}

\begin{abstract}
This study investigated the suitability of epoxidized vegetable oils as additives in place of aromatic processing aids and activators in natural rubber based truck tyre tread compounds. The processability and cure characteristics of the compounds and physical properties of the vulcanizates were compared with those of the standard vulcanizate, which was prepared with a standard mineral oil -Dutrex R. Epoxidation of the oils was carried out (formed in situ) using the peracetic acid method and epoxidation was confirmed using ${ }^{1} \mathrm{H}$ NMR and ${ }^{13} \mathrm{C}$ NMR spectra. Epoxidized vegetable oils (EVO) were used at a fixed dose in the compounding process as processing aids. These oils were used in the compounding process to ascertain the possibility of using them as activators and processing aids.
\end{abstract}

Epoxidized palm oil (EPO) showed better processing properties, polymer filler interaction and dispersion properties and best heat resistance ability. Epoxidized sunflower oil (ESFO) can be used as an accelerator with the combination of sulphenamide to overcome the problem of reversion. Epoxidized soya bean oil (ESBO), EPO and ESFO were better alternative processing aids than petroleum based aromatic oils, which have been reported as carcinogenic. The cure characteristics and physical properties of the vulcanizate based on EPO suggested that this oil could be used as an activator in rubber compounding.

Keywords: Activator, epoxidation, palm oil, processing aid, soya bean oil, sunflower oil.

\section{INTRODUCTION}

High aromatic oils, which originate as derivatives of the petrochemical industry, have been widely used traditionally as extender oils for rubber compounding. Their popularity is due to good compatibility with most of the common natural and synthetic elastomers. Extender oils are often referred to as process or softening oils and are added to rubber compounds in the production process of rubber articles to achieve acceptable processability. They are also essential to ensure the technical performance of tyres, in particular for their road adherence. High aromatic oils have a high content of polycyclic aromatic hydrocarbons (PAH), which are suspect carcinogens. On the $13^{\text {th }}$ of February 2004, Council of the European Communities (2005) adopted a proposal aimed at banning the use and marketing of PAH-rich extender oils from the $1^{\text {st }}$ of January 2010. However, the ban is not fully enforced to date since effective alternatives are not available in the market.

Vegetable oils are environment friendly, biodegradable, less costly and readily available. The use of these oils in many applications has attracted the attention of many researchers due to their potential as substitutes to petrochemical derivatives. Modification and application of vegetable oils as additives in polymer processing have been reported (Okieimen et al., 2005; Jayewardhana et al., 2009). Epoxidized vegetable oils (EVO) can be used as processing aids for polymers (Kuriakose \& Varghese, 2003; Dasgupta et al., 2007). The unsaturated double bonds of vegetable oils rich in oleic, linoleic and linolenic acyl groups may be converted into the more reactive oxirane moiety by reaction with peracids or peroxides, which have formed in situ (Kirk-Othmer, 1968; Padmasiri et al., 2009). Compaired to virgin vegetable oils EVOs contain epoxy rings in their chemical structure and could behave in a more similar manner to PAH-rich extender oils. Previous work using palm, soya bean and sunflower oils as alternative processing aids and activators in carbon black filled natural rubber has demonstrated the possibility of using some vegetable oils as processing aids (Jayewardhana et al., 2009). 
Studies of eco-friendly processing aids for rubber compounds have been reported (Dasgupta et al., 2007). The naturally occurring oils have reported to show better processing properties, polymer filler interaction and dispersion properties (Jayewardhana et al., 2009). Naturally occurring oils have been found to be suitable alternatives to the presently available, comparatively more costly low PAH oils in the market in the form of mild extract solvates (MES), treated distillate aromatic extracts (TDAE) and naphthenic oils.

It has been reported that replacement of aromatic oils with rice bran oil (RBO) and epoxidized rice bran oil (ERBO) does not adversely affect the physical properties of the carbon black filled natural rubber / polychloroprene blends. RBO contains a good amount of free fatty acids; hence it has been reported to act as a co-activator in addition to its role as a processing aid (Kuriakose \& Varghese, 2003). The use of non toxic and readily available vegetable oils in place of petroleum based aromatic oils has been demonstrated. Various oils (vegetable oil, paraffinic oil and castor oil) at a fixed dose (1phr) have been used and it has been found that the vegetable oils exhibit enhanced properties in comparison to those of paraffinic oil (Kukreja et al., 2003).

Since a previous study has revealed that some virgin vegetable oils are capable of replacing PAH containing extenders without any adverse effects to the vulcanizates (Jayewardhana et al., 2009), it is interesting to ascertain the effect of epoxy derivatives of these oils as processing aids and accelerators. Hence, the main objective of this research project is to investigate the chemical interaction of epoxidized vegetable oils (EVO) with natural rubber (NR) in order to find out their suitability as processing aids and as activators.

\section{METHODS AND MATERIALS}

Vegetable oils: The three vegetable oils used were palm oil (Ngo Chew Hong Edible Oil Pvt. Ltd., Singapore), soya bean oil (Ngo Chew Hong Edible Oil Pvt. Ltd., Singapore) and sunflower oil (Sime Darby Edible Products Ltd., Singapore). They were used without any further purification.

Aromatic oil: Industrial grade processing aid Dutrex R obtained from the local market was used as the standard mineral oil (NMO).

Fourier transform infrared spectroscopy (FT-IR) analysis of pure oils: Neat liquid method was followed to obtain the FT-IR spectra of the oils. Two drops of pure oil was placed on the center of a clean, dry $\mathrm{KBr}$ plate using a Pasteur pipette. The second $\mathrm{KBr}$ plate was placed on top of that plate and both were compressed together. The two drops of oil were evenly spread between the two plates as a thin film. Both plates were mounted on a sample holder and it was placed in the infrared beam of the spectrometer. The spectra were recorded in the range of 4000-500 $\mathrm{cm}^{-1}$ using a FT-IR spectrometer (Model 320 FT-IR / Thermo Necolet AVATAR ${ }^{\text {TM}}$ ).

Iodine value test for pure oils: Samples of palm oil (713.4 mg), soya bean oil $(212.3 \mathrm{mg})$ and sunflower oil $(203.4 \mathrm{mg})$ were weighed into 3 different reagent bottles. The oils were dissolved in $15.0 \mathrm{~cm}^{3}$ of carbon tetrachloride. Exactly $25.00 \mathrm{~cm}^{3}$ of Wij's solution (BDH Chemicals,UK) was added to each reagent bottle and closed. The bottles were shaken gently and kept in the dark for $1 \mathrm{~h}$. Exactly $20.00 \mathrm{~cm}^{3}$ of $15 \% \mathrm{KI}$ solution (Avonchem Ltd., UK) was added to each bottle after $1 \mathrm{~h}$. About $100 \mathrm{~cm}^{3}$ of distilled water was added and the mixture was titrated with standardized $0.1 \mathrm{M} \mathrm{Na} \mathrm{S}_{2} \mathrm{O}_{3}$ solution (Loba Chemie Pvt. Ltd., India) with constant and vigorous shaking until the yellow colour of the titrant almost disappeared. A few drops of starch indicator was added to the flask and titrating was continued until the blue colour of the mixture became colourless. The volume of $0.1 \mathrm{M} \mathrm{Na} \mathrm{S}_{2} \mathrm{O}_{3}$ consumed for the titration was recorded (Horwitz, 2005). The procedure was carried out in duplicate for palm oil, soya bean oil and sunflower oil separately. Blank tests were carried out along with each oil sample.

Epoxidation using resin as a catalyst: The extent of unsaturation was calculated as a molarity, considering the iodine value of the respective oil. The amount of reagent that should be added to the reactor was calculated according to the molar ratio, where the process involved mixing of oil containing $1.0 \mathrm{~mol}$ of unsaturation with 0.55 mol of glacial acetic acid (Loba Chemie Pvt. Ltd., India), 12\% dry resin (Amberlite / IR 120) based on the weight of the oil and $1.1 \mathrm{~mol}$ of $\mathrm{H}_{2} \mathrm{O}_{2}$ (East Anglian Chemicals, UK).

The two liquid phases were separated from the resin catalyst by decantation using a separation funnel. The separated oil phase was dissolved in $30 \mathrm{~cm}^{3}$ of diethyl ether (Loba Chemie Pvt. Ltd., India), anhydrous $\mathrm{Na}_{2} \mathrm{SO}_{4}$ (Loba Chemie Pvt. Ltd., India) was added and kept for about $1 \mathrm{~h}$. The clear solution was separated by decantation and the diethyl ether was removed by rotary evaporation. Finally the viscous liquid of epoxide was obtained (KirkOthmer, 1968; Vogel , 1973; Padmasiri et al., 2009).

FT-IR analysis: FT-IR for the epoxide was carried out according to the liquid method described previously. 
${ }^{1} \mathrm{H}$ NMR and ${ }^{13} \mathrm{C}$ NMR analysis: About $25 \mathrm{mg}$ of the sample oil was dissolved in $\mathrm{CDCl}_{3}$ solvent. The solution was placed in the NMR sample tube and the tube was sealed. Then it was placed in the NMR instrument. The ${ }^{1} \mathrm{H}$ NMR and ${ }^{13} \mathrm{C}$ NMR (Mercury -300BB “chem.-300”) spectra were recorded.

Determination of extent of epoxidation: The extent of epoxidation was calculated using the percentage reduction of iodine value.

Sample preparation: The natural rubber (RSS) was masticated in an internal mixer (Baker Perkins Engineers, London, UK) for about $3 \mathrm{~min}$. The antioxidant-Nisopropyl-N'-phenyl-p-phenylenediamine (IPPD) was added and milling was carried out for about $1 \mathrm{~min}$. Carbon black (N330) and processing oil were next mixed and the mixture was blended for about $2 \mathrm{~min}$.

Zinc oxide $(\mathrm{ZnO})$ and stearic acid were mixed together and added to the mixture. After proper mixing, the mixture was cooled under ambient conditions. The mixture was then introduced to the two roll mill and the mixing was carried out again. Sulphur and Ncyclohexylbenzthiazylsulphenamide (CBS) (accelerator) were added together to the two roll mill. Proper mixing was achieved by several mill passes for about $2 \mathrm{~min}$. By adjusting the nip between the rolls, a thin compounded rubber sheet was obtained.

Table 1: Tyre tread formulations used to evaluate suitability of epoxidized oils as a processing aid (boxed area represents the proposed replacements for processing oil)

\begin{tabular}{lcccc}
\hline & \multicolumn{4}{c}{ Formulation } \\
Materials & $\mathrm{F}_{\mathrm{NMO}}$ & $\mathrm{F}_{\mathrm{EPO}}$ & $\mathrm{F}_{\mathrm{ESBO}}$ & $\mathrm{F}_{\mathrm{ESFO}}$ \\
\hline Natural rubber (RSS)/phr & 100 & 100 & 100 & 100 \\
ZnO (Rubber grade)/phr & 5.0 & 5.0 & 5.0 & 5.0 \\
Stearic acid/phr & 2.0 & 2.0 & 2.0 & 2.0 \\
Carbon black (HAF & 50 & 50 & 50 & 50 \\
N330)/phr & & & & \\
Dutrex R/phr & 5.0 & 0.0 & 0.0 & 0.0 \\
EPO/phr & 0.0 & 5.0 & 0.0 & 0.0 \\
ESBO/phr & 0.0 & 0.0 & 5.0 & 0.0 \\
ESFO/phr & 0.0 & 0.0 & 0.0 & 5.0 \\
IPPD (N-isopropyl- N'- & 1.5 & 1.5 & 1.5 & 1.5 \\
phenyl-p-phenylenediamine)/phr & & & & \\
Sulphur (Rhombic)/phr & 2.5 & 2.5 & 2.5 & 2.5 \\
CBS (N-cyclohexyl & 1.0 & 1.0 & 1.0 & 1.0 \\
& & & &
\end{tabular}

benzthiazylsulphenamide)/phr
The above process was carried out with epoxidized palm oil (EPO), epoxidized soya bean oil (ESBO), epoxidized sunflower oil (ESFO) and Dutrex R aromatic oil. As given in Table 1 , the formulation labeled $\mathrm{F}_{\mathrm{NMO}}$ was prepared by using Dutrex $\mathrm{R}$ as the standard processing aid, the three formulations labeled $\mathrm{F}_{\mathrm{EPO}}, \mathrm{F}_{\mathrm{ESBO}}$ and $\mathrm{F}_{\mathrm{ESFO}}$ were prepared replacing Dutrex R with EPO, ESBO and ESFO to study the effect of EVOs as processing oils. The formulations in Table 2 were used to study the effect of EVOs as processing aids and activators in place of aromatic processing oil and stearic acid. In Table 2, the first column under formulation is labeled $\mathrm{F}_{\mathrm{NMO} / \mathrm{WSA}}$ and contains Dutrex $\mathrm{R}$ as both the processing oil and the activator (replacing stearic acid). Columns 2,3 \& 4 under formulation in Table 2 labelled $\mathrm{F}_{\mathrm{EPO} / \mathrm{WSA}}, \mathrm{F}_{\mathrm{ESBO} / \mathrm{WSA}}$ and $\mathrm{F}_{\mathrm{ESFO} / \mathrm{WSA}}$ have been prepared replacing both Dutrex $\mathrm{R}$ (5.0 phr) and stearic acid (2.0 phr) with EPO, ESBO and ESFO, respectively.

Molding: The molding of rubber sheets was carried out using a laboratory hydraulic press (Yeji Corporation, Taiwan) at a temperature of $150{ }^{\circ} \mathrm{C}$ and a pressure of $20 \mathrm{MPa}$.

Evaluation of cure characteristics: Curing tests were performed using a moving die rheometer (MDR) (Ekron Terk, Taiwan) at $1500{ }^{\circ} \mathrm{C}$.

Table 2: Tyre tread formulation used to evaluate suitability of epoxidized oils as a processing aid and an activator (boxed area represents the proposed replacements for processing oil and activator)

\begin{tabular}{|c|c|c|c|c|}
\hline \multirow[b]{2}{*}{ Materials } & \multicolumn{3}{|c|}{ Formulation } & \multirow[b]{2}{*}{$\mathrm{F}_{\mathrm{ESFO} / \mathrm{WSA}}$} \\
\hline & $\mathrm{F}_{\mathrm{NMO} / \mathrm{WSA}^{*}}$ & $\mathrm{~F}_{\mathrm{EPO} / \mathrm{WSA}}$ & $\mathrm{F}_{\mathrm{ESBO} / \mathrm{WSA}}$ & \\
\hline Natural rubber (RSS)/phr & 100 & 100 & 100 & 100 \\
\hline $\mathrm{ZnO}$ (Rubber grade)/phr & 5.0 & 5.0 & 5.0 & 5.0 \\
\hline Carbon black (HAF & 50 & 50 & 50 & 50 \\
\hline \multicolumn{5}{|l|}{ N 330)/phr } \\
\hline Dutrex R/phr & 7.0 & 0.0 & 0.0 & 0.0 \\
\hline $\mathrm{EPO} / \mathrm{phr}$ & 0.0 & 7.0 & 0.0 & 0.0 \\
\hline $\mathrm{ESBO} / \mathrm{phr}$ & 0.0 & 0.0 & 7.0 & 0.0 \\
\hline $\mathrm{ESFO} / \mathrm{phr}$ & 0.0 & 0.0 & 0.0 & 7.0 \\
\hline $\begin{array}{l}\text { IPPD (N-isopropyl- N'- } \\
\text { phenyl-p- } \\
\text { phenylenediamine)/phr }\end{array}$ & 1.5 & 1.5 & 1.5 & 1.5 \\
\hline Sulphur (Rhombic)/phr & 2.5 & 2.5 & 2.5 & 2.5 \\
\hline $\begin{array}{l}\text { CBS (N-cyclohexyl } \\
\text { benzthiazylsulphenamide) }\end{array}$ & 1.0 & 1.0 & 1.0 & 1.0 \\
\hline$/ \mathrm{phr}$ & & & & \\
\hline
\end{tabular}




\section{Evaluation of physical properties:}

a) tensile strength: Dumbbell shaped test pieces were punched out from the molded sheets. The thickness of the test pieces were measured with a bench thickness gauge (Wallace Instruments, UK). The test pieces were tested using a Hounsfield testing machine (Model 100R - UK) according to ISO $37: 1994(\mathrm{E})$ at $250{ }^{\circ} \mathrm{C}$ and at a cross head speed of $500 \mathrm{~mm} / \mathrm{min}$.

b) tear strength: Crescent shaped test pieces were punched out from the molded sheets. The thickness of the test pieces were measured using a bench thickness gauge and tested according to ISO 34-1:1994 (E) at $250{ }^{\circ} \mathrm{C}$ and at a cross head speed of $500 \mathrm{~mm} / \mathrm{min}$.

c) hardness: Hardness was tested using a Digi test hardness tester (Wallace Instruments, UK) according to ISO 48:1994(E).

d) rebound resilience: Test pieces were tested on Lüpke resilience tester (Wallace Instruments, UK) according to ISO 4662-1986 (E).

e) abrasion mass loss \%: DIN abrasion tester (model APH-40, Hampden, Northampton, UK) was used according to DIN 53516 in order to test the abrasion mass loss.

f) compression set: Initial thickness of the test pieces were recorded. The test pieces were compressed for $72 \mathrm{~h}$ at room temperature and thickness of the test pieces after compression [using compression set apparatus (Wallace Instruments, UK)] was also recorded. Percentage compression set was calculated according to the equation given in ISO 815:1991 (E).

g) swelling index: Initial weights of the test pieces were recorded and the specimens were immersed in glass bottles containing toluene. These bottles were covered to minimize the evaporation of toluene. The samples were kept for few days and weighed regularly until a constant weight was achieved.

h) aging studies: Tensile and tear strength were evaluated after aging. Aging was done in an air circulating oven at $100{ }^{\circ} \mathrm{C}$ (Sanyo Gallenkamp, UK) for $22 \mathrm{~h}$ according to ISO 37:1994(E) and ISO 34-1:1994 (E).

\section{RESULTS}

\section{Iodine values of oils}

Iodine values of palm, soya bean and sunflower oils were 53, $124 \& 126$ respectively. Although these natural oils were commercial products obtained from the local market, the iodine values are in between the range of recorded values given in literature (O'Brein, 1998). The iodine values of oils were used for the determination of the number of double bonds present in a unit volume of oil. Epoxidations were carried out according to the iodine value data, which indicate the degree of unsaturation in oils. Further, the iodine values of epoxidized vegetable oils were used in the determination of the percentage epoxidation.

\section{Epoxidation}

The epoxidized products that were obtained were lighter in colour and higher in viscosity compared to the pure oils.

\section{Confirmation of epoxide}

Asymmetrical stretching of the $(\mathrm{C}-\mathrm{C})$ in the epoxy ring during the contraction of the $(\mathrm{C}-\mathrm{O})$ bond at $950-810 \mathrm{~cm}^{-1}$ can be observed (Figure 1). (C-H) stretching of epoxy ring at 3050-2990 $\mathrm{cm}^{-1}$ and epoxy ring bonds stretching and contracting inphase near $1250 \mathrm{~cm}^{-1}$ cannot be observed clearly due to the overlapping with other peaks (Pavia, 1979; Silverstine, 2005).

\section{DISCUSSION}

Recent research has shown the suitability of natural oils as processing aids and activators (Kuriakose \& Rajendran, 1994; Ismail et al., 1997; Kuriakose \& Varghese, 2003; Dasgupta et al., 2007; Jayewardhana et al., 2009).

\section{Confirmation of epoxidation}

According to the FT-IR results, there is some evidence that epoxidation has occurred. However, the formation of epoxide could not be confirmed clearly using the peaks that appeared in the spectra. Therefore ${ }^{1} \mathrm{H}$ NMR and ${ }^{13} \mathrm{C}$ NMR analysis were carried out to confirm epoxidation.

\section{${ }^{1} \mathrm{H}$ NMR and ${ }^{13} \mathrm{C}$ NMR analysis}

The ${ }^{1} \mathrm{H}$ NMR spectrum for epoxidized soya bean oil is shown in Figure 2. The epoxy proton was observed at 3.0-3.1 ppm as given in literature, which results from the splitting caused by cis-epoxide (Karunanayake \& Fernando, 2006). Further, the ${ }^{13} \mathrm{C}$ NMR spectrum given in Figure 3 shows a signal between 53-60 ppm as given in literature (Kemp, 1986; Karunanayake \& Fernando, 2006), which results from the di-substituted epoxy ring carbon of the epoxidized soya bean oil. According to 


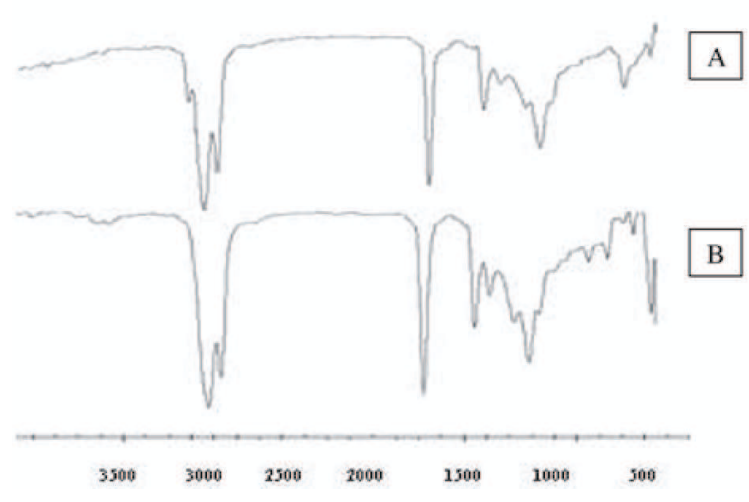

Figure 1: FTIR spectra of SBO (A), ESBO (B)

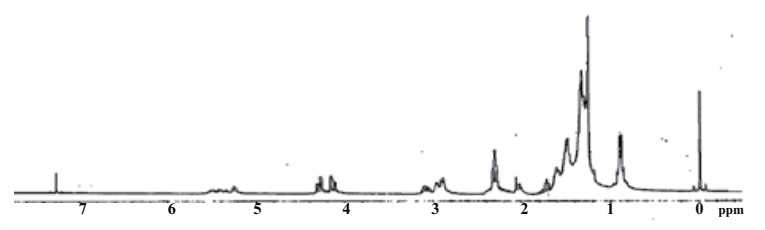

Figure 2: ${ }^{1} \mathrm{H}$ NMR spectrum of epoxidized soyabean oil

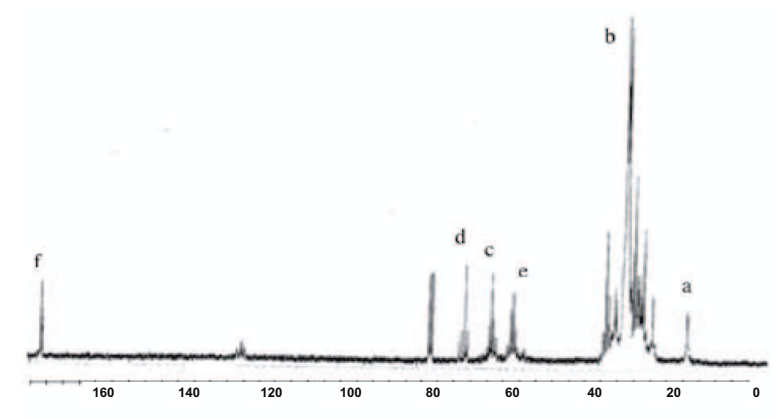

Figure 3: ${ }^{13} \mathrm{C}$ NMR spectrum of epoxidized soyabean oil

the NMR spectral data, the formation of epoxidized derivatives of the oils during the epoxidation reaction can be confirmed.

\section{Epoxidized vegetable oils as processing aids}

Processing safety is determined by the time that elapses before the onset of cure. In tyre tread preparation, there must be sufficient delay or scorch resistance to permit shaping, forming and flowing in the mold, which facilitate the grip of the tyre. The results show that scorch time values are slightly lower for the compounds prepared with epoxidized vegetable oils when compared with those prepared with aromatic oils (Table 3). Cure time

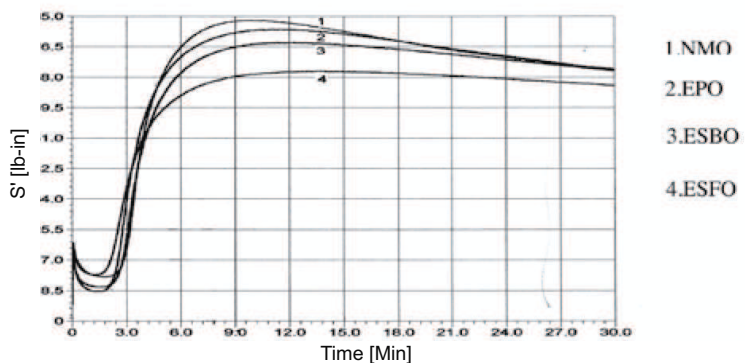

Figure 4: Rheographs for compounds prepared using different processing aids

of the compound containing EPO is similar to that of the compound containing NMO. The former shows the highest cross link density at $\mathrm{t}_{90}$, indicating the ability for energy conservation in processing of rubber similar to the NMO. However, this relationship cannot be observed for the compound containing NMO and EPO (Table 4).

The swelling index is an indication of cross link density, which in turn correlates with the physical properties of the various vulcanizates. Hence, delta cure value, which is a measure of the cross-link density should be related to swelling index. Delta cure increases with the increase of cross link density. Compounds containing EPO has the highest cross link density compared to other natural oils studied (Table 3 ). Swelling test measurements support this observation (Table 4). According to the results the compound containing EPO shows good swelling resistance and the highest cross link density. Presence of epoxy groups instead of unsaturation, facilitate the polarpolar interaction with carbon black. Therefore, coupling action is greater with EVOs than the natural vegetable oils (Jayewardhana et al., 2009). Generally, vegetable oils are unsaturated long chain fatty esters. Hence, the polar esters as well as unsaturated esters can interact

Table 3: Cure characteristics of vulcanizates containing EVOs

\begin{tabular}{llllll}
\hline $\begin{array}{l}\text { Processing } \\
\text { aid }\end{array}$ & $\begin{array}{l}\text { Scorch } \\
\text { time/ } \\
\text { min. }\end{array}$ & $\begin{array}{l}\text { Cure } \\
\text { time/ } \\
\text { min. }\end{array}$ & $\begin{array}{l}\text { Cure rate } \\
\text { index } \\
\text { value/ } \mathrm{min}^{-1}\end{array}$ & $\begin{array}{l}\text { Maximum } \\
\text { torque } \\
\text { value/ Nm }\end{array}$ & $\begin{array}{l}\text { Delta } \\
\text { cure } \\
\mathrm{Nm}\end{array}$ \\
\hline NMO & 2.60 & 6.09 & 28.65 & 9.48 & 8.09 \\
EPO & 2.12 & 6.09 & 25.19 & 9.20 & 8.27 \\
ESBO & 2.38 & 6.41 & 24.81 & 8.78 & 7.71 \\
ESFO & 1.93 & 6.39 & 22.42 & 7.88 & 6.42 \\
NMO/WSA & 2.81 & 6.50 & 27.12 & 7.48 & 6.30 \\
EPO /WSA & 2.47 & 5.66 & 31.32 & 7.03 & 5.95 \\
ESBO /WSA & 2.70 & 5.16 & 40.62 & 5.77 & 4.72 \\
ESFO/WSA & 2.55 & 5.37 & 35.49 & 5.95 & 5.00 \\
\hline
\end{tabular}

*Without stearic acid 
Table 4: Physical properties of vulcanizates containing EVOs

\begin{tabular}{|c|c|c|c|c|c|c|c|c|}
\hline Property & $\mathrm{NMO}$ & EPO & ESBO & ESFO & NMO/WSA & EPO/WSA & ESBO/WSA & ESFO/WSA \\
\hline $\begin{array}{l}\text { Tensile strength } \\
\text { (MPa) BA }\end{array}$ & 25.95 & 26.97 & 25.80 & 26.83 & 27.30 & 29.18 & 27.36 & 28.65 \\
\hline $\begin{array}{l}\text { Tensile strength } \\
\text { (MPa) AA }\end{array}$ & 19.41 & 21.31 & 19.80 & 19.80 & 26.40 & 22.32 & 22.00 & 22.02 \\
\hline $\begin{array}{l}\text { Tear strength } \\
\left(\mathrm{N} \mathrm{mm}^{-1}\right) \mathrm{BA}\end{array}$ & 166.5 & 172.3 & 156.3 & 169.3 & 155.6 & 154.2 & 155.8 & 148.2 \\
\hline $\begin{array}{l}\text { Tear strength } \\
\left(\mathrm{N} \mathrm{mm}^{-1}\right) \mathrm{AA}\end{array}$ & 118.3 & 139.8 & 118.3 & 122.5 & 151.1 & 136.3 & 110.1 & 120.0 \\
\hline $\begin{array}{l}\% \text { Retention of } \\
\text { tensile strength }\end{array}$ & 75 & 79 & 77 & 74 & 97 & 76 & 80 & 77 \\
\hline $\begin{array}{l}\% \text { Retention of } \\
\text { tear strength }\end{array}$ & 71 & 81 & 76 & 72 & 97 & 88 & 71 & 81 \\
\hline $\begin{array}{l}\text { Hardness } \\
\text { (IRHD units) }\end{array}$ & 63.5 & 65.1 & 59.2 & 58.8 & 42.4 & 42.3 & 37.3 & 33.7 \\
\hline $\begin{array}{l}\text { Modulus at } \\
300 \% \text { elongation } \\
\text { (MPa) }\end{array}$ & 13.5 & 14.9 & 13.4 & 12.4 & 5.2 & 5.6 & 5.0 & 4.6 \\
\hline $\begin{array}{l}\text { Elongation at } \\
\text { break \% }\end{array}$ & 502.2 & 489.8 & 518.5 & 518.4 & 655.1 & 664.2 & 648.4 & 696.5 \\
\hline $\begin{array}{l}\text { Rebound resilience } \\
\text { (\%) }\end{array}$ & 48.7 & 49.2 & 51.3 & 50.3 & 62.0 & 65.0 & 60.7 & 62.0 \\
\hline $\begin{array}{l}\text { Abrasion mass } \\
\text { loss } \%\end{array}$ & 9.8 & 9.2 & 9.6 & 9.7 & 12.4 & 14.0 & 15.2 & 15.0 \\
\hline $\begin{array}{l}\text { Compression } \\
\text { set } \%\end{array}$ & 13.42 & 13.80 & 11.83 & 14.92 & 11.66 & 8.95 & 7.14 & 15.24 \\
\hline Swelling Index & 1.56 & 1.58 & 1.76 & 1.87 & 2.20 & 2.44 & 2.69 & 2.60 \\
\hline
\end{tabular}

BA - Before aging; AA - After aging

properly with the carbon black surface, which consists of active polar groups while its non polar paraffin chain end could interact with the rubber molecule. High unsaturations are responsible for the increment of cross link density, and the double bonds present in oil are subject to degradation easily. This can be overcome by epoxidation of natural oils, which improves the polarity. When the oil is undergoing coupling action, if it is highly polar in nature, there would be a restriction to interact with natural rubber as it is less polar in nature. Therefore, the coupling action reduces when it is highly epoxidized. Thus, it can be inferred that EPO, which has the lowest level of epoxidation, gives better characteristics over ESBO and ESFO (Table 5).

According to the rheographs, it is clear that all graphs show a reversion, but compound containing
ESFO has the lowest level of reversion among them. In compounding, a conventional vulcanizing (CV) system was used and generally $\mathrm{CV}$ systems lead to reversion. Crosslink (predominantly polysulphidic) density of the compound containing ESFO is higher than that of the compound containing ESBO, and ESFO shows a minimum reversion. This indicates that ESFO acts as an activator leading to a minimum reversion.

Table 5: Properties of EVOs

\begin{tabular}{lccc}
\hline Type of oil & EPO & ESFO & ESBO \\
\hline Iodine value & 7 & 13 & 12 \\
$\begin{array}{l}\text { Extent of } \\
\text { epoxidation, } \%\end{array}$ & 87 & 89 & 91 \\
\hline
\end{tabular}

Journal of the National Science Foundation of Sri Lanka 39 (3) 


\section{Physical properties}

Degree of cross linking and the type of cross link play a significant role on tensile strength and tear strength of vulcanizates. The highest tensile strength was achieved for the vulcanizate containing EPO followed by the vulcanizate containing ESFO. In the tyre tread formulation the accelerator (CBS) to sulphur ratio is $1: 2$ and hence the vulcanizing system is conventional. In conventional vulcanizing $(\mathrm{CV})$ systems, the relative proportions of cross link types are polysulphidic 70\%, disulphidic $15 \%$ and monosulphidic $15 \%$ (Kukreja et al., 2003; Jayewardhana et al., 2009). The conventional vulcanization system produces a higher number of polysulphidic linkages. The vulcanizate containing ESFO has lesser cross link density but its tensile and tear strength are little higher than that of the vulcanizates containing NMO and ESBO. This is probably due to the presence of more polysulphidic linkages; in the former, which is indicated by the highest reversion. Better retention values for tensile and tear strength were obtained for the vulcanizates containing EPO and ESBO than the standard oil. Therefore, after aging, properties were found to be better for vulcanizates prepared with EVOs, because of the presence of epoxy groups in the oil instead of unsaturations (Krik-Othmer, 1968). Tensile strength and tear strength reduce after aging due to the breakage of predominately polysulphidic linkages at elevated temperatures.

Variation of elongation at break is generally similar to that of tensile strength. Hence, elongation at break of EPO is expected to be the highest. However, due to coupling action, reinforcing fillers are highly bound to the elastomer chains. Even though a higher stress is applied the entanglements present in elastomers prevent freedom to move and cannot attain a tension flow. Therefore elongation at break reduces. According to literature (Dasgupta et al., 2007), better dispersibility of filler can be attained by NMO; hence elongation at break of this is lower than that of ESBO and ESFO. EPO shows an acceptable hardness value when compared with the other two epoxidized natural oils. The results reveal that hardness varies with cross link density.

The ratio of the energy returned to the energy applied is termed as the resilience. When the deformation is an indentation due to a single impact, this ratio is termed the "rebound resilience".

If the elasticity is higher, then less deformation energy is dissipated as heat. Heat buildup is the measure of the amount of impressed energy that is absorbed. Even though the EPO containing compound has a higher cross link density, the rebound resilience is low. This is probably due to the C-black reinforcement of rubber that hinders the elasticity of the vulcanizate and hence the energy is dissipated as heat (Kundu, 2000; Kundu \& Kukreja, 2002). Higher rebound resilience values of ESBO and ESFO compared to EPO and NMO indicate the ability of their molecules to store energy. The abrasion mass loss is considerably lesser in EPO containing compounds than in the others due to greater dispersion of C-black with EPO according to the theory of coupling action (Kundu \& Kukreja, 2002). In filled compounds, abrasion mechanism is by roll formation. This roll formation is greater in vulcanizates with higher elongation at break values. Therefore, abrasion mass loss results are in agreement with elongation at break values.

\section{Epoxidized vegetable oils as activators}

Scorch time is higher for NMO and for ESBO, respectively. Therefore they show better processing safety than EPO and ESFO.

The lowest scorch time is obtained for EPO. This means that the generation of cross links starts quickly in the compound containing EPO and reveals that the contribution of EVO as the activator for vulcanization rate is highest in EPO followed by ESFO.

In rubber compounding, activators are used to increase the rate of vulcanization by activating the accelerator, so that it performs more effectively. They are generally high molecular weight monobasic acids or mixtures of stearic, oleic, lauric, palmitic and myristic acids. The effect of palm oil fatty acids on cure characteristics and mechanical properties of carbon black filled natural rubber compounds has been reported (Kukreja et al., 2003; Jayewardhana et al., 2009). NMO has the highest cure time and lower cure rate index. Thus, the energy consumption for the vulcanizing process would be highest when compared with EVOs. Therefore EVOs are superior to standard aromatic oils in case of energy conservation when they are used as activators.

\section{Physical properties}

NMO showed the highest cross link density, even though it has the lowest tensile strength. EVOs showed much higher tensile strengths, indicating plasticization over NMO when used as activators. EPO showed higher rebound resilience. Earlier, when EVOs were used as processing aids, EPO showed the least rebound resilience due to the coupling action. But in this case it had actively participated in the activation process with the accelerator and sulphur to attain a highly cross linked 
vulcanizate. Therefore, the formed vulcanizate has less filler-elastomer interaction in comparison to NMO. Thus, elastomer molecules have the ability to store the energy and in turn impart higher rebound resilience.

\section{CONCLUSION}

Processing safety of the vulcanizates containing EVOs is lower than that of the Dutrex $\mathrm{R}$ aromatic oil. EPO has the highest ability to attain maximum cross link density compared to other oils. Dutrex R vulcanizate shows the highest cure rate index compared to the vulcanizates containing EVOs. ESFO has the ability to act as an accelerator and can work with sulfenamide in a synergistic way by minimizing the reversion of the rheographs. EVOs exhibit lower cure times compared to that of Dutrex $\mathrm{R}$ and shows the ability to conserve energy during processing. EPO shows lowest scorch time, indicating the generation of cross links and greater activator role than the others. In keeping with the results, EVOs have higher activating ability. Cure characteristics and the physical properties of the vulcanizate based on EPO suggest the suitability of it as an activator.

Hence, it can be concluded that:

1. Epoxidized soya bean oil (ESBO), epoxidized palm oil (EPO) and epoxidized sun flower oils (ESFO) are better alternative processing aids compared to petroleum based aromatic oils, which have been reported to be carcinogenic.

2. EPO could be used as an activator in rubber compounding.

\section{REFERENCES}

1. Council of the European Communities (2005). The approximation of laws, regulations and administrative provisions relating to the classification, packaging and labeling of dangerous substances; amending for the $27^{\text {th }}$ July 1976, Council Directive 76/769/EEC.

2. Dasgupta S., Agrawal S.L., Bandyopadhyay S., Chakraborty S., Muhoopadhyay R., Malkani R.K. \& Ameta S.C. (2007). Characterization of eco-friendly processing aids for rubber compound. Polymer Testing 26(4): 489-500.

3. Horwitz W. (2005). Official Methods of Analysis of AOAC International, $17^{\text {th }}$ edition, pp. 7-9. AOAC international, Maryland, USA.

4. Ismail H., Salmiah I. \& Tsukahara Y. (1997). Palm oil fatty acid as an activator in carbon black filled natural rubber compounds: Effect of vulcanization system. Polymer International 44(4): 523-529.

5. Jayewardhana W.G.D, Perera G.M., Edirisinghe D.G. \& Karunanayake L. (2009). Study on natural oils as alternative processing aids and activators in carbon black filled natural rubber. Journal of the National Science Foundation of Sri Lanka 37(3): 187-193.

6. Karunanayake L. \& Fernando P.N.J. (2006). Effect of incorporation of peanut and sesame oils and their epoxides on the structure of poly vinyl chloride. Journal of the National Science Fondation of Sri Lanka 34(2): 97-102

7. Kemp W. (1986). NMR in Chemistry: A Multinuclear Introduction, pp. 72-73, 211-213. Macmillan Education Ltd., Oxford, USA.

8. Kirk-Othmer (1968). Encyclopedia of Chemical Technology, $2^{\text {nd }}$ edition, pp. 239-256. John Wiley and Sons, Inc., New York, USA.

9. Kukreja T.R., Chauhan R.C., Choe S. \& Kundu P.P. (2003). Effect of the doses and nature of vegetable oil on carbon black / rubber interactions: studies on castor oil and other vegetable oils. Journal of Applied Polymer Science 87(10): 1574-1578.

10. Kundu P.P. (2000). Improvement of filler-rubber interaction by the coupling action of vegetable oil in carbon black reinforced rubber. Journal of Applied Polymer Science 75(6): 735-739.

11. Kundu P.P. \& Kukreja T.R. (2002). Surface modification of carbon black by vegetable oil-its effect on the rheometric, hardness, abrasion, rebound resilience, tensile, tear, and adhesion properties. Journal of Applied Polymer Science 84(2): 256-260.

12. Kuriakose A.P. \& Rajendran G. (1994). Rice bran oil as a novel compounding ingredient in sulphur vulcanization of natural rubber. European Polymer Journal 31(6): 595-602.

13. Kuriakose A.P. \& Varghese M. (2003). Use of rice bran oil and epoxidized rice bran oil in carbon black filled natural rubber polychloroprene blends. Journal of Applied Polymer Science 90(14): 4084-4092.

14. O'Brien R.D. (1998). Fats and Oils Formulating and Processing for Applications, pp. 9-25. Technomic Publishing Company, Inc., Basel, Switzerland.

15. Okieimen F.E., Pavithran C. \& Bakare I.O. (2005). Epoxidation and hydroxylation of rubber seed oil: one-pot multi-step reactions. European Journal of Lipid Science Technology 107(5): 330-336.

16. Padmasiri K. G., O’Brien M. \& Karunanayake L. (2009). Epoxidation of some vegetable oils and their hydrolyzed products with peroxyformic acid - optimized to industrial scale. Journal of the National Science Foundation of Sri Lanka 37(4): 229-240.

17. Pavia D.L., Lampman G.M. \& Kriz G.S. (1979). Introduction to Spectroscopy: A Guide for Students of Organic Chemistry, pp. 26-38. W.B. Saunders Company, London, UK.

18. Silverstine R.M., Basler G.C. \& Morril T.C. (2005). Spectrometric Identification of Organic Compounds, $5^{\text {th }}$ edition, pp. 105-121, 171-254. John Willey and Sons, Inc., New York, USA.

19. Vogel I. (1973). A Text Book of Quantitative Inorganic Analysis Including Elementary Instrumental Analysis, $3^{\text {rd }}$ edition, pp. 362-378, 702-712. Longman Publishers Ltd., London, UK. 